\title{
5
}

\section{COMPORTAMIENTO PRODUCTIVO DE CLONES DE CAFÉ ROBUSTA (COFFEA CANEPHORA P) EN MANGLARALTO, ECUADOR.}

Mercedes Arzube, Néstor Orrala, Ángel León, Lenni

Ramírez. 


\title{
Comportamiento productivo de clones de café robusta (Coffea Canephora p) en Manglaralto, Ecuador.
}

\author{
Production behavior of robusta coffee \\ (Coffea Canephora p) clones in Manglaralto, Ecuador.
}

\author{
Mercedes Arzube Mayorga, Néstor Orrala Borbor, Ángel León Mejía, \\ Lenni Ramírez Flores \\ Centro de Investigaciones Agropecuarias \\ Facultad de Ciencias Agrarias \\ Universidad Estatal Península de Santa Elena Campus \\ marzube@upse.edu.ec
}

\begin{abstract}
Resumen
La investigación se realizó en la granja experimental Manglaralto, propiedad de la Universidad Estatal Península de Santa Elena, ubicada en las coordenadas UTM 528944m E y 9796468m S zona 17M datum WGS84 a 12 msnm, con una topografía inferior al 1\%, la investigación tuvo como objetivo determinar el comportamiento productivo de clones de café robusta (Coffea canephora P.), de alta productividad en las condiciones agroecológicas, de Manglaralto Ecuador. Para el establecimiento del ensayo, se utilizaron 23 clones de café robusta, seleccionados por COFENAC en la región amazónica del norte de Ecuador. Los clones fueron dispuestos al azar, cada clon es una unidad experimental representada por 20 plantas, sembradas a una distancia de $3 \times 3$ metros. Los resultados preliminares se sometieron al análisis de estadística descriptiva, determinándose medidas de tendencia central y de dispersión media aritmética, desviación estándar, coeficiente de variación, entre clones y dentro de los clones. Sin embargo, al cuarto año, se destacan como promisorios en producción los clones 1, 4, 5, 6, 14, 15, 16 y 18. El comportamiento productivo es muy alentador considerando que el clon 1 obtuvo producción de 61 quintales y el clon 15 alcanzo 39,3 quintales de café oro por hectárea, los demás clones enunciados obtienen producción promedio de 42 quintales.
\end{abstract}

Palabras claves: Clones, café robusta, café oro, productividad

\begin{abstract}
The research was carried out in the experimental farm Manglaralto, owned by the Peninsula State University of Santa Elena, located at the coordinates UTM 528944m E and 9796468m S zone 17M datum WGS84 at $12 \mathrm{msnm}$, with topography of less than 1\%, research (Coffea canephora P.), high productivity in the agroecological conditions of Manglaralto Ecuador. For the establishment of the trial, 23 clones of robust coffee, selected by COFENAC in the Amazon region of northern Ecuador, were used. The clones were arranged randomly, each clone is an experimental unit represented by 20 plants, planted at a distance of $3 \times 3$ meters. Preliminary results were submitted to the descriptive statistics analysis, determining measures of central tendency and mean arithmetic dispersion, standard deviation, coefficient of variation, between clones and within the clones. However, in the fourth year, clones 1, 4, 5, 6, 14, 15, 16 and 18 stand out as promising in production. The productive behavior is very encouraging considering that clone 1 obtained production of 61 quintals and the clone 15 reached 39.3 quintals of gold coffee per hectare, the other clones enunciated obtain average production of 42 quintals.
\end{abstract}

Key words: Clons, robusta coffee, gold coffee, productivity. 


\section{Introducción}

Según el (INAMI, 2009) la península de Santa Elena posee una variedad de microclimas que puede ser aprovechada con la introducción de cultivos. Hace décadas, la zona era productora de café, especialmente en las estribaciones de la cordillera Chongón Colonche (Ministerio de Agricultura, 2010).

La caficultura actividad fue desapareciendo con el inicio de la explotación petrolera, lo que trajo consigo, la deforestación y posterior desertificación $\mathrm{y}$ salinización de los suelos. Investigación relacionada con los sistemas de producción, tiene como base el conocimiento de los factores (exógenos y endógenos) que intervienen en los mismos, como una necesidad obligada para el desarrollo de alternativas de gestión (Castaldoet al., 2003).

El litoral ecuatoriano tiene zonas con condiciones agroecológicas adecuadas para la producción de café robusta, en las cuales se puede establecer plantaciones de este cultivo, para abastecer a la industria local y su exportación contribuyendo así, a la creación de empleo y mejora de ingresos a la cadena cafetalera (Corral, 2013)

Actualmente, la provincia de Santa Elena goza de ventajas comparativas como la existencia de 50.000 ha. bajo la influencia del trasvase Daule-Peripa(Ortiz, F. 2008); en la península de Santa Elena se obtienen varias oportunidades y ventajas en la producción agrícola, la más clara es que no está sujeta a la estacionalidad en la producción, que por su latitud y clima que otros países no tienen (Gerrero, 2009); hay evidencias campesinas de que en el valle del río Javita y en la zona de la norte de la provincia se podría cultivar café robusta(Ortiz, 2008).

Bajo este criterio, la adaptación de clones de café robusta se convierte en la primera etapa de la selección de clones productivos que en el mediano plazo permitirán la diversificación agro-productiva y como mecanismo para dinamizar la economía campesina.

\section{Materiales y métodos}

La investigación se ejecutó en la granja experimental Manglaralto de la Universidad Estatal Península de Santa Elena, ubicada en las coordenadas UTM $528944 \mathrm{~m}$ E y $9796468 \mathrm{~m} \mathrm{~S}$ zona 17 Mdatum WGS-84 a 12 msnm, topografía menor al 1\%.

En la zona se presentan dos temporadas bien diferenciadas: la época de invierno que va de diciembre a abril; y, la temporada de garúa que se ubica en los meses de mayo a noviembre, con precipitación promedio anual de $200 \mathrm{~mm}$; por otra parte, la temperatura fluctúa entre los 16 a 30 grados centígrados en determinados meses del año, y la heliófila 12 horas por día (Natura \& P, 1995).

El comportamiento climático (Tabla 1) correspondiente a la temperatura, precipitación y humedad relativa en Manglaralto.

Tabla 1. Datos climáticos de Manglaralto

\begin{tabular}{lccc}
\hline Meses & $\begin{array}{c}\text { Tempera- } \\
\text { tura }\end{array}$ & Precipitación & $\begin{array}{c}\text { Humedad } \\
\text { relativa }\end{array}$ \\
\hline Enero & 26 & 130 & 82 \\
Febrero & 26 & 226 & 77 \\
Marzo & 28 & 93 & 81 \\
Abril & 28 & 17 & 84 \\
Mayo & 28 & 4 & 85 \\
Junio & 26 & 1 & 71 \\
Julio & 24 & 0 & 53 \\
Agosto & 23 & 3 & 71 \\
Septiembre & 23 & 3 & 72 \\
Octubre & 23 & 9 & 87 \\
Noviembre & 24, & 0 & 82 \\
Diciembre & 25 & 0 & 86 \\
\hline
\end{tabular}

Fuente: CENAIM - ESPOL San Pedro. 2013.

Los niveles de fertilidad del suelo y de calidad agua se determinaron mediante el análisis de laboratorio mostrando $\mathrm{pH} 7,7 ; \mathrm{MO} 4,4 \%$; Nitrógeno 37 medio; P 65 A, K 4,92, Ca 20,8 A, Mg 3,7 A, S14M, Zn 2,6 B, Cu 5,6 A, Fe 22 M, Mn 4,6 B y B 0,86 A. Suma de bases $29,53 \mathrm{meq} / 100$ gramos, C.E. $0,82 \mathrm{~ms} / \mathrm{cm}$.

El agua de riego extraída de pozo presenta una clase C3S1 (aguas de salinidad media alta y de contenido bajo de sodio).

Para el establecimiento del banco de Germoplasma, se utilizan 26 clones (Tabla 2), materiales seleccionados por COFENAC en la región amazónica del norte de Ecuador.

Los clones fueron dispuestos al azar. Cada clon es una unidad experimental representada por 20 plantas, sembradas a una distancia de 3 × 3 metros.

Los resultados preliminares se sometieron al análisis de estadística descriptiva, determinándose medidas de tendencia central y de dispersión media aritmética, desviación estándar, coeficiente de variación, entre clones y dentro de los clones.

Por ser un experimento, el control de malezas se realizó en forma manual; en cuanto al control fitosanitario fue de acuerdo a la incidencia de las mismas, se utilizó productos amigables con el ambiente. A más de las labores agro técnicas necesarias para determinar necesidades de riego, control fitosanitario, etc., se realizaron las labores de cosecha 
La tabla 2 detalla los clones de café robusta que se encuentran en estudio en el banco de germoplasma del Centro de Prácticas Manglaralto.

Tabla 2. Clones del banco de germoplasma en Manglaralto.

\begin{tabular}{lll}
\hline No & CLON & $\begin{array}{c}\text { CÓDIGO } \\
\text { COFENAC- } \\
\text { UPSE }\end{array}$ \\
\hline $\mathbf{1}$ & RP-S-004 & C SE -01 \\
$\mathbf{2}$ & RP-S-007 & C SE -02 \\
$\mathbf{3}$ & RP-S-009 & C SE -03 \\
$\mathbf{4}$ & RP-S-013 & C SE -04 \\
$\mathbf{5}$ & RP-S-015 & C SE -05 \\
$\mathbf{6}$ & RP-S-018 & C SE -06 \\
$\mathbf{7}$ & PCH-AU-10 & C SE -07 \\
$\mathbf{8}$ & FA-AU-015 & C SE -08 \\
$\mathbf{9}$ & BF-AU-02 & C SE -09 \\
$\mathbf{1 0}$ & BF-AU-04 & C SE -10 \\
$\mathbf{1 1}$ & BA-L-02 & C SE -11 \\
$\mathbf{1 2}$ & JR-O-01 & C SE -12 \\
$\mathbf{1 3}$ & PA-O-02 & C SE -13 \\
$\mathbf{1 4}$ & NP-2044 & C SE -14 \\
$\mathbf{1 5}$ & NP-2024 & C SE -15 \\
$\mathbf{1 6}$ & NP-4024 & C SE -16 \\
$\mathbf{1 7}$ & NP-3056 & C SE -17 \\
$\mathbf{1 8}$ & SA-BC-016 & C SE -18 \\
$\mathbf{1 9}$ & MN-BC-019 & C SE -19 \\
$\mathbf{2 0}$ & LY-BC-021 & C SE -20 \\
$\mathbf{2 1}$ & NP-3013 & C SE -21 \\
$\mathbf{2 2}$ & NP-3018 & C SE -22 \\
$\mathbf{2 3}$ & NP-3072 & C SE -23 \\
\hline
\end{tabular}

Las variables experimentales evaluadas fueron las siguientes:

Variables agronómicas: se evaluaron con una frecuencia mensual: altura de la planta medida en centímetros $(\mathrm{cm})$, desde el suelo hasta el ápice del tallo principal, usando una regla graduada, diámetro de tallo medido a $20 \mathrm{~cm}$ sobre el nivel del suelo utilizando un calibrador "Vernier", en milímetros, diámetro de copa usando una regla graduada, considerando la rama bajera más larga del cafeto, en centímetros.

Variables productivas: rendimiento $\left(\mathrm{t} / \mathrm{ha}^{-1}\right)$, la cosecha se realizó todos los meses y la producción se expresó en quintales de café cereza por hectárea y año; para ello se sumó cada una de las cosechas realizadas.

\section{Resultados y Discusión}

Los resultados de producción de café robusta al cuarto año de edad del cultivo de los 23 clones en estudio, se muestran en la figura 3. Sin embargo, los clones que se destacan como promisorios son los clones $1,4,5,6,14,16$ y 18 .
El comportamiento productivo es muy alentador considerando que el clon 1 obtuvo producción de 61 quintales de café oro por hectárea, los demás clones enunciados obtienen producción promedio de 4,2 t/ha${ }^{1}$. En el oriente ecuatoriano. (INIAP, 2010) manifiesta que a partir de una evaluacion amplia de café robusta (Coffea canephora) proveniente de Costa Rica, la EECA seleccionó clones que presentaron alta produccion que se ubicaron en un promedio de 6,4 $\mathrm{t} / \mathrm{ha}^{-1}$ de café oro, resultados similares alcanzaron Gaskin; Macías; y Mejías (2013), en la variedad Caturra.

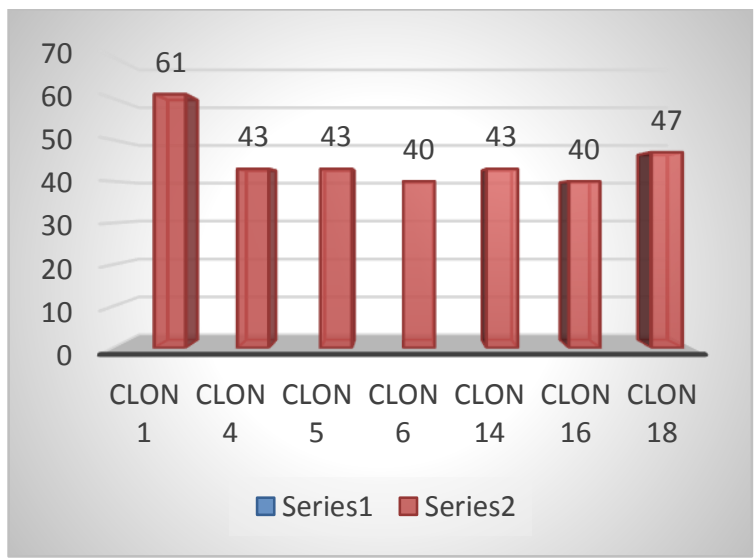

Figura 3. Producción de café oro al cuarto año del cultivo.

La altura de la planta de café es importante ya que nos muestra el crecimiento ortotrópico de la planta, lo que va a proporcionar mandolina que garantizaran la producción en los próximos años coincidiendo con autores como (Blanco, Haggar, \& Moraga, 2003); Macías (2008).

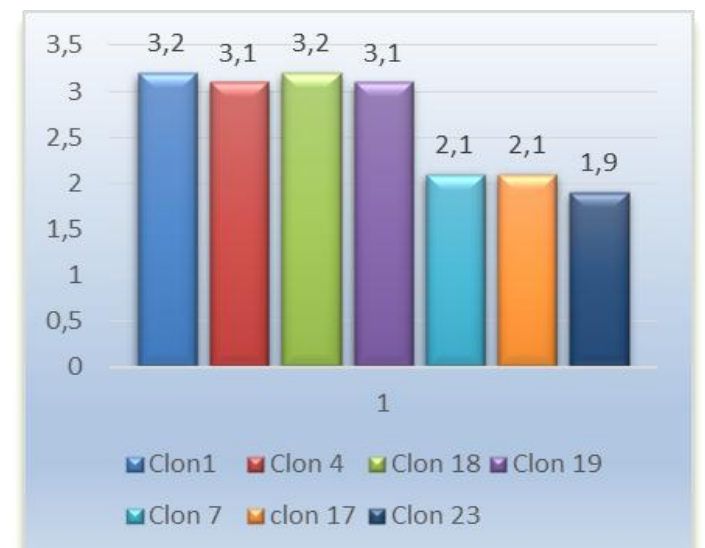

Figura 2. Altura de plantas de café robusta al cuarto año del cultivo.

Analizando el comportamiento de la altura de las plantas se observa que los clones $1,4,18$ y 19 alcanzaron los mayores promedios, ubicándose los 
rangos entre $3,20,3,10, \quad 3,2 \quad 3,10$ metros, respectivamente; las menores alturas obtuvieron los clones 7, 17 y 23 con promedios de 2,10, 2,10 y 1,9 metros respectivamente (Figura 2). Estos datos muestran similitud con lo encontrado por (Plaza, Rey, \& Duicela, 2015), ellos mencionaron que la variable agronómica altura de planta, es utilizada como medio de selección de plantas de café, igualmente se evidenció plantas que alcanzaron porte bajo con un promedio de $220 \mathrm{~cm}$, entre los materiales de porte medio alcanzaron una altura de $300 \mathrm{~cm}$, y porte alto $301 \mathrm{~cm}$, igualmente Gaskin; Macías; y Mejías (2013) plantean que la altura es un factor determinante en el desarrollo y posterior cosecha de la cereza.

\section{CONCLUSIONES}

1. La altura promedio de los clones promisorios en producción se encuentra en 3,10 metros.

2. De los clones considerados como promisorios destacan en producción el clon 1 con $6,1 \mathrm{t} / \mathrm{ha}^{-1}$ seguido de clon 18 con $4,7 \mathrm{t} / \mathrm{ha}^{-1}$ de café oro por año.

3. Los resultados de la investigación muestran que se podría considerar los clones 1, 4, 5, 6, 14, 16, 18 que podrían ser utilizados para formar un policlon.

4. Con las premisas de los resultados se continúa con la investigación que tiene carácter regional, observando con rigurosidad el manejo agrotécnico, que ensayos de esta naturaleza exigen.

\section{Agradecimiento}

A las autoridades de la Universidad Estatal Península de Santa Elena, y Facultad de Ciencias Agrarias por el apoyo financiero al desarrollo de esta investigación.

\section{Referencias}

[1] Blanco, M., Haggar, J., \& Moraga, P. (2003). Morfología de café (Coffea arábica L.), en lotes comerciales. Nicaragua. Agronomía Mesoamericana, 100.

[2] Castaldo, A., Cruz, A. d., A, M., Martos, J., Pamio, J., \& Mendoza. (2003). Caracterización de la invernada en el nordeste de la provincia de La Pampa. XXIV Reunión Anual de la Asociación Argentina de Economía. Argentina: Asociación Argentina de Economía.
[3] Corral, L. (2013). Sembrado Desiertos. La Deuda Social y Ecológica generada por el endeudamiento externo en el Proyecto de Propósito Múltiple "Jaime Roldós Aguilera", Acción Ecológica, Segunda ed. Quito.

[4] Guerrero. (2009). Estudio del potencial agroindustrial y de exportación para la producción de pepino en la península de Santa Elena y los recursos necesarios para su implantación. 3.

[5] INAMI. (2009). Base de datos Estación meteorológica UPSE. Santa Elena - Ecuador: INSTITUTO NACIONAL DE METEOROLOGÍA E HIDROLOGÍA.

[6] INIAP. (2010). Contribuciones del INIAP a la región Amazónica Ecuatoriana. QuitoEcuador: Estación Experimental Central de la Amazonia: 2da edición.

[7] Ministerio de Agricultura, G. A. (2010). Tercer Censo Nacional Agropecuario. Resultados Nacionales y Provinciales. Quito-Ecuador: INEC-MAG-SICA.

[8] Natura, F., \& P, A. (1995). Desarrollo sostenible: Aproximaciones conceptuales. Quito, Ecuador: UICN - Fundación Natura.

[9] Ortiz, F. (2008). Potencialidades de la Península de Santa Elena. Subsecretaría de Inclusión económica.

[10] Plaza, L., Rey, \& Duicela. (2015). Caracterización Fenotípica del Germoplasma de Coffea canephora Pierre Base Para S su mejoramiento en Ecuador. EPAMCIENCIAS, 10.

[11] Gaskin B; Macías, I; Mejías J (2013). Determinación de las características dimensionales y distribución de los cafetos variedad Caturra Rojo en condiciones de ladera. Revista Ciencias Técnicas Agropecuarias.vol.22 no.3: ISSN: 2071-0054

[12] Macías I (2008). Determinación de las propiedades físico-mecánicas y dimensionales del café (variedad Caturra Rojo y Caturra Amarillo y su influencia en la selectividad de la cosecha. Tesis presentada en opción al grado de Doctor. Universidad Politécnica de Madrid. 
Disponible en:

[http://www.upm.es/observatorio/vi/index.jsp?p

ageac $=$ actividad.jsp\&id_actividad $=54006]$.

Consultado [12 de febrero de 2017]. 